Radiative mechanism to light fermion masses in the MSSM

This article has been downloaded from IOPscience. Please scroll down to see the full text article.

JHEP01(2008)072

(http://iopscience.iop.org/1126-6708/2008/01/072)

View the table of contents for this issue, or go to the journal homepage for more

Download details:

IP Address: 200.19.254.173

The article was downloaded on 31/08/2011 at 16:23

Please note that terms and conditions apply. 


\title{
Radiative mechanism to light fermion masses in the MSSM
}

\author{
C.M. Maekawa and M.C. Rodriguez \\ Fundação Universidade Federal do Rio Grande-FURG, Departamento de Fúsica, \\ Av. Itália, km 8, Campus Carreiros 96201-900, Rio Grande, RS Brazil \\ E-mail: dfscmm@furg.br, mcrodriguez@einstein.fisica.furg.br
}

ABSTRACT: In a previous work we applied a discrete symmetry $\left(\mathcal{Z}_{2}^{\prime}\right)$ in order to light fermions acquire mass only at one loop level. This symmetry and the assumption of alignment between fermions and sfermions allow us to avoid FCNC problems. Here a more general hypothesis of flavor mixing in the sfermion sector of MSSM is considered and we show that the $s$ quark is heavier than $u, d$ quarks due to different content of sfermions contributions. Our results are in agreement with the experimental constraint on the values of sfermions masses.

KEYWORDs: Supersymmetry Phenomenology, Supersymmetric Effective Theories. 


\section{Contents}

1. Introduction 1

2. $\mathcal{Z}_{2}^{\prime}$ symmetry in the MSSM

3. Masses of the supersymmetric particles 5

3.1 Super-CKM basis for Sfermions

3.1.1 The squarks

3.1.2 The masses of selectrons 10

3.2 The masses of gluinos 10

4. The mechanism of mass generation 10

4.1 Light fermion masses 10

4.2 Final expressions 13

5. Conclusions 13

A. Notation 14

A.1 The fields of MSSM 14

B. Interaction of fermion-sfermion-gauginos 15

G. SPA convention 16

D. Feynman integration 16

\section{Introduction}

Family problems of elementary particles have shown to be a challenge since one realizes that the strong interaction respects isospin, lepton and baryon numbers conservation. Due to excess of baryons over antibaryons in our universe the baryon number conservation were pointed to be broken [1]. Later Weinberg points to lepton and baryon numbers conservations do not need to be a prior assumption in the framework of Grand Unified Theories (GUT) where the processes are mediated by superheavy particles with mass $M \simeq$ $10^{14} \mathrm{GeV}$ [2]. However the non observation of proton decay [3], Electric Dipole Moment of elementary particles 4 , and neutron-antineutron oscillations [5] points to a non trivial violation mechanism of these symmetries.

To this puzzle one can joint questions about the mass generation mechanism which are able to describe the observed mass hierarchy of particles and mixing angles. A known 
mechanism is based on Yukawa couplings between fermions and scalars of the models (Standard Model (SM), Supersymmetric models (SUSY) and GUT) but among the possibilities of such couplings there are sources for dangerous Flavor Change Neutral Currents (FCNC) processes, like proton decay and neutron-antineutron oscillation.

Besides, the recent data from neutrinos experiments add more questions: What is their mass scale? Which is their mass organization pattern?

The SM describes the family structure as doubletes of $S U(2)[6]$ and it has been able to described most of present day data. In the case of mass generation mechanism, SM tells us that fermions obtain their masses through Yukawa couplings with Higgs doublet while neutrinos have no mass. However the values of these couplings constants remain arbitrary. There are also other aspects which cannot be explain in the framework of SM, e.g., the non-leptonic without strangeness changing processes. In this case the problem is not due to family structure, it is the interplay between strong and week interaction. The strong repulsive core keeps the nucleons away from each other at a distance enough to prevent the gauge bosons exchanges between quarks [7]. In this case one has to deal with nucleons and pions degrees of freedom instead of quarks and gluons. A reliable and consistent description with underline principles is obtained in the framework of Chiral Perturbation Theory (ChPT) [8].

In order to determine the values of Yukawa coupling constants or at least to find a way to constrain them there are approaches based on GUT $+\mathrm{SUSY}+G_{f}$ [9] or SM $+G_{f}$ [10] where $G_{f}$ is an additional family symmetry required in order to constrain these constants. On GUT + SUSY $+G_{f}$ approaches, the masses of fermions are degenerate at GUT scale and a mass generation mechanism based on renormalization group equations gives rise to the hierarchy pattern observed at Fermi scale. One classifies quarks as $u$ type $(t, c, u)$ and $d$ type $(b, s, d)$ and the hierarchy pattern follows a power law, e.g.: for horizontal hierarchy:

$$
\begin{array}{ll}
m_{t}: m_{c}: m_{u} \sim 1: \varepsilon_{u}: \varepsilon_{u}^{2} & \varepsilon_{u} \simeq 500^{-1} \\
m_{b}: m_{s}: m_{d} \sim 1: \varepsilon_{d}: \varepsilon_{d}^{2} & \varepsilon_{d} \simeq 50^{-1} \\
m_{\tau}: m_{\mu}: m_{\varepsilon} \sim 1: \varepsilon_{e}: \varepsilon_{e}^{2} & \varepsilon_{e} \simeq 50^{-1}
\end{array}
$$

where $m_{u}$ and $m_{d}$ are the current quark mass.

Another source of flavor problems is the misalignment of fermion- sfermion couplings. It is due to the transformation that diagonalizes the fermion mass matrix does not simultaneously diagonalize the corresponding sfermion mass squared matrices. The lack of observation of the decays $\mu \rightarrow e \gamma, \tau \rightarrow \mu \gamma$ and $\tau \rightarrow e \gamma$ put some constraints on the leptonslepton coupling. On the other hand, processes like $b \rightarrow s \gamma$ decay and the measurements of mass difference in $B^{0} \bar{B}^{0}$ and $D^{0} \bar{D}^{0}$ yield constraints on the quark-squark couplings, the most stringent restrictions here come from one knows about $K^{0} \bar{K}^{0}$ mixing.

In general there are three ways to suppress this problem [11, 12]:

(a) Arrange for degeneracy or universality of masses of sfermions with the same quantum numbers. In this scenario the $K^{0} \bar{K}^{0}$ mixing expression is suppressed because the $\Delta m_{\tilde{d}_{\imath}}$ is very small; 
(b) One can assume an alignment between the fermion and sfermion mass matrices so that both can be made diagonal in the same basis. In this case, the fermion and sfermion mass matrices is said to be aligned. Such an alignment is included in models with so-called horizontal symmetries which links the various generations;

(c) The third choice is to take the masses of sfermions of the first two generations to be very large, in the multi-TeV range. This solution to the SUSY flavor problem is known as decoupling.

One may also consider various combinations of these options. The flavor violating contributions have been parametrized by Gabbiani et al [13] in the framework of mass insertion method. In this approach one works in a basis where the mass matrix of fermions of a given charge as well as the corresponding fermion-sfermion-neutral gaugino couplings are diagonal in flavor space. Flavor violation is then described by flavor non-diagonal entries and the constraints are expressed as bounds on the dimensionless quantities.

The first attempt to apply the radiative mechanism of mass generation to the light fermions was suggested by S. Weinberg [14, 15. Later L. Ibañez shows if SUSY is spontaneously broken one generates only tiny small fermion masses radiatively [16]. In order to restrict this mechanism to the first family a discrete symmetry is applied into SUSY models in refs. [17, 18]. From the analysis performed by Ferrandis [19, 20] the radiative mechanism of mass generation of fermions is allowed through sfermion-gaugino loops and the observed flavor physics is obtained if "the supersymmetry breaking terms receive small corrections, which violate the symmetry of the superpotential".

In a previous work [21] we followed a mass pattern of Chiral descriptions [8] where a Chiral scale $\left(\Lambda_{\chi}=1 \mathrm{GeV}\right)$ allow us to classify the quarks as light ones $(u, d$ and $s)$ and heavy ones $(c, t$ and $b)$. Thus we introduced a $\mathcal{Z}_{2}^{\prime}$ symmetry in the MSSM and in the SUSY Left-Right Models in order to allow the light quarks acquire mass only by means of radiative mass generation mechanism [17, 18, 22] while the FCNC problems are avoided - The Chiral mass hierarchy pattern and a consistent picture with experimental data of Cabibbo-Kobayashi-Maskawa (CKM) matrix were obtained. We also showed that under $\mathcal{Z}_{2}^{\prime}$ symmetry, a similar pattern for electron, muon and tau can be obtained. The heavy leptons ( $\mu$ and $\tau$ ) acquire mass at tree level while the electron acquires mass at 1-loop level - We also assumed the alignment of squarks with the quarks and due to the absence of inter family mixing of squarks each quark receives contribution only from its corresponding supersymmetric partner and in order to describe the mass gap between strange and non strange quarks we need to consider the strange supersymmetric partner heavier than nonstrange supersymmetric partners.

In this work we remove the assumption of alignment between quark and squarks sectors and we explore the effects of $\mathcal{Z}_{2}^{\prime}$ symmetry $^{1}$ on the masses of sfermions in section 3 . In the section 1 the mass of light fermions are re-evaluated and the contribution of sfermions are still different to each mass of light fermions.

\footnotetext{
${ }^{1}$ We review this symmetry in section 2
} 
We present at section 4.2 the masses of the light fermions. From these results, we can explain why the quarks $u$ and $d$ are lighter than the $s$ quark. Our notation is shown in the appendix A. The details of computations are presented in our appendices B D.

\section{2. $\mathcal{Z}_{2}^{\prime}$ symmetry in the MSSM}

In our previous work, we introduced the following $\mathcal{Z}_{2}^{\prime}$ symmetry on the Lagrangian of the MSSM 21]

$$
\widehat{d}_{2 L}^{c} \longrightarrow-\widehat{d}_{2 L}^{c}, \quad \widehat{d}_{3 L}^{c} \longrightarrow-\widehat{d}_{3 L}^{c}, \widehat{u}_{3 L}^{c} \longrightarrow-\widehat{u}_{3 L}^{c}, \widehat{l}_{3 L}^{c} \longrightarrow-\widehat{l}_{3 L}^{c},
$$

while the others superfields of the model $^{2}$ are even under this symmetry.

The invariant superpotential under $\mathcal{Z}_{2}^{\prime}$ and $R$-parity symmetries is given by

$$
W_{\mathrm{R}-\mathrm{inv}}^{\mathcal{Z}_{2}^{\prime} \mathrm{even}}=\mu \hat{H}_{1} \hat{H}_{2}+\sum_{\imath=1}^{3} y_{\imath 1}^{d} \hat{Q}_{\imath L} \hat{H}_{1} \hat{d}_{1 L}^{c}+\sum_{\imath=1}^{3} \sum_{\jmath=1}^{2} y_{\imath \jmath}^{l} \hat{L}_{\imath L} \hat{H}_{1} \hat{l}_{\jmath L}^{c}+\sum_{\imath=1}^{3} \sum_{\jmath=1}^{2} y_{\imath \jmath}^{u} \hat{Q}_{\imath L} \hat{H}_{2} \hat{u}_{\jmath L}^{c}
$$

The $R$-parity symmetric but $\mathcal{Z}_{2}^{\prime}$ forbidden terms are given by:

$$
W_{\mathrm{R}-\text { inv }}^{\mathcal{Z}_{2}^{\prime} \text { odd }}=\sum_{\imath=1}^{3} \sum_{\jmath=2}^{3} y_{\imath \jmath}^{d} \hat{Q}_{\imath L} \hat{H}_{1} \hat{d}_{\jmath L}^{c}+\sum_{\imath=1}^{3} y_{\imath 3}^{l} \hat{L}_{\imath L} \hat{H}_{1} \hat{l}_{3 L}^{c}+\sum_{\imath=1}^{3} y_{\imath 3}^{u} \hat{Q}_{\imath L} \hat{H}_{2} \hat{u}_{3 L}^{c} .
$$

As a consequence of eq. (2.2), the fermions $u, d, s$ and $e$ are prevented to acquire mass at tree level in a way similar as presented in refs. 17-20]. These fermions are massless due to the absence of the terms showed in eq. (2.3). On the other hand, Supersymmetric non-renormalization theorem guarantee that corrections to the fermions masses are very small, even if the discrete symmetry (2.1) is broken.

An interesting question we don't deal in our first work is: How Does our $\mathcal{Z}_{2}^{\prime}$ symmetry act on the sfermion sector? The answer can be obtained from eq. (2.1). The behavior of scalar components of chiral superfields under $\mathcal{Z}_{2}^{\prime}$ symmetry are given by:

$$
\begin{array}{llll}
\tilde{d}_{2}^{c} \longrightarrow-\tilde{d}_{2}^{c}, & d_{2}^{c} \longrightarrow-d_{2}^{c}, & \tilde{d}_{3}^{c} \longrightarrow-\tilde{d}_{3}^{c}, & d_{3}^{c} \longrightarrow-d_{3}^{c}, \\
\tilde{u}_{3}^{c} \longrightarrow-\tilde{u}_{3}^{c}, & u_{3}^{c} \longrightarrow-u_{3}^{c}, & \tilde{l}_{3}^{c} \longrightarrow-\tilde{l}_{3}^{c}, & l_{3}^{c} \longrightarrow-l_{3}^{c},
\end{array}
$$

while all other fields of the model are even. It worth noting that the $\mathcal{Z}_{2}^{\prime}$ symmetry has the same role as in the fermion sector: it forbids the flavor mixing between the third family and the other two families of sfermions.

As we show below, we also obtain the following features: because the couplings between the squarks from the third family with the other two families are forbidden, the assumption of alignment between quark and squark sector can be removed. Then a particular texture for mass matrix of squarks consistent with physical bounds comes out. Therefore the $\mathcal{Z}_{2}^{\prime}$ symmetry help us to keep under control the dangerous FCNC problems and we still obtain the mass hierarchy pattern without any additional assumptions.

\footnotetext{
${ }^{2}$ Our notation and the particles contents of this model are shown in appendix A.
} 
Another feature in the $\mathcal{Z}_{2}^{\prime}$ symmetric case is the null value for EDM of electron and of neutron. Because of the symmetry the left-right mixing angle vanished in the sleptons and squarks sectors. These mixing angles contribute to the EDM calculation and in this case there are no contributions to the EDM coming from the MSSM. Therefore, the only contribution to the EDM of these particles come from SM.

\section{Masses of the supersymmetric particles}

The discussion in this section is based on review articles of refs. [11, 12, 23- 25]. We start with a general study of mass generation of supersymmetric particles. The reason to perform this study is due to the fact that masses and mixing of sparticles are of crucial importance both experimentally and theoretically: i) they determine the properties of the sparticles searched for and ii) they are directly related to the question of how SUSY is broken [11, 12].

Once $\mathrm{SU}(2)_{L} \otimes \mathrm{U}(1)_{Y}$ symmetry is broken, fields with the same $\mathrm{SU}(3)_{c} \otimes \mathrm{U}(1)_{\mathrm{em}}$ quantum numbers (and, of course, R-parity, $\mathcal{Z}_{2}^{\prime}$ and spin) can mix with each other. In the framework of Standard Model, $B^{0}$ and $W^{i}$ mix to $\gamma, Z^{0}$, and $W^{ \pm}$is an example of this kind of mixing. Also the Dirac masses of quarks and leptons can be understood as such mixing terms. For the case of MSSM, this mixing also affects squarks, sleptons, Higgs bosons, as well as gauginos and higgsinos. The only exception is the gluino, which is the only color octet fermion in the model.

\subsection{Super-CKM basis for Sfermions}

There is no longer alignment assumption and we perform the diagonalization procedure in the Super-CKM (SCKM) basis. Here we present the relevant equations for our work and a detailed discussion can be found at ref. [26].

Likewise we have done in our superpotential, ${ }^{3}$ we separate the soft SUSY breaking terms into two terms:

$$
\mathcal{L}_{\text {soft }}=\mathcal{L}_{\text {soft }}^{\mathcal{Z}_{2}^{\prime} \text { even }}+\mathcal{L}_{\text {soft }}^{\mathcal{Z}_{2}^{\prime} \text { odd }}
$$

where $\mathcal{L}_{\text {soft }}^{\mathcal{Z}_{2}^{\prime} \text { even }}$ is the even component under $\mathcal{Z}_{2}^{\prime}$ (eq. (2.4)) and it reads:

$$
\begin{aligned}
\mathcal{L}_{\text {soft }}^{\mathcal{Z}_{2}^{\prime} \text { even }}=-\frac{1}{2}\left(\sum_{\imath=1}^{8}\right. & \left.m_{\tilde{g}} \lambda_{C}^{\imath} \lambda_{C}^{2}+\sum_{p=1}^{3} m_{\lambda} \lambda_{A}^{p} \lambda_{A}^{p}+m^{\prime} \lambda_{B} \lambda_{B}+h . c .\right)-\left[\sum_{\imath=1}^{3} \sum_{\jmath=1}^{3} \tilde{L}_{\imath L}^{\star}\left(M_{L}^{2}\right)_{\imath \jmath} \tilde{L}_{\jmath L}\right. \\
& +\sum_{\imath=1}^{2} \sum_{\jmath=1}^{2} \tilde{l}_{\imath L}^{c \star}\left(M_{l}^{2}\right)_{\imath \jmath} \tilde{l}_{\jmath L}^{c}+\tilde{l}_{3 L}^{c \star}\left(M_{l}^{2}\right)_{33} \tilde{l}_{3 L}^{c}+\sum_{\imath=1}^{3} \sum_{\jmath=1}^{3} \tilde{Q}_{\imath L}^{\star}\left(M_{Q}^{2}\right)_{\imath \jmath} \tilde{Q}_{\jmath L} \\
& +\sum_{\imath=1}^{2} \sum_{\jmath=1}^{2} \tilde{u}_{\imath L}^{c \star}\left(M_{u}^{2}\right)_{\imath \jmath} \tilde{u}_{\jmath L}^{c}+\tilde{u}_{3 L}^{c \star}\left(M_{u}^{2}\right)_{33} \tilde{u}_{3 L}^{c}+\tilde{d}_{1 L}^{c \star}\left(M_{d}^{2}\right)_{11} \tilde{d}_{1 L}^{c}
\end{aligned}
$$

\footnotetext{
${ }^{3}$ See eqs. (2.2), (2.3)
} 


$$
\begin{aligned}
& \left.+\sum_{\imath=2}^{3} \sum_{\jmath=2}^{3} \tilde{d}_{\imath L}^{c \star}\left(M_{d}^{2}\right)_{\imath \jmath} \tilde{d}_{\jmath L}^{c}\right]-M_{1}^{2} \tilde{H}_{1}^{\star} \tilde{H}_{1}-M_{2}^{2} \tilde{H}_{2}^{\star} \tilde{H}_{2}-M_{12}^{2}\left(H_{1} H_{2}+\text { h.c. }\right) \\
& -\left[\sum_{\imath=1}^{3} \sum_{\jmath=1}^{2} H_{1} \tilde{L}_{\imath L}\left(A^{l}\right)_{\imath \jmath} \tilde{l}_{\jmath L}^{c}+\sum_{\imath=1}^{3} \sum_{\jmath=1}^{2} H_{2} \tilde{Q}_{\imath L}\left(A^{u}\right)_{\imath \jmath} \tilde{u}_{\jmath L}^{c}\right. \\
& \left.\quad+\sum_{\imath=1}^{3} H_{1} \tilde{Q}_{\imath L}\left(A^{d}\right)_{\imath 1} \tilde{d}_{1 L}^{c}+\text { h.c. }\right]
\end{aligned}
$$

The $m^{\prime}, m_{\lambda}$, and $m_{\tilde{g}}$ are $\mathrm{U}(1), \mathrm{SU}(2)$ and $\mathrm{SU}(3)$ gaugino masses respectively. The mass terms of Higgs fields are denoted by $M_{1}^{2}, M_{2}^{2}$, and $M_{12}^{2}$. The symbol (*) in a scalar field is the charge conjugate of this field, it means we take their anti-particle.

The components whose also break $\mathcal{Z}_{2}^{\prime}$ symmetry $\left(\mathcal{L}_{\text {soft }}^{\mathcal{Z}_{2}^{\prime} \text { odd }}\right)$ are given by

$$
\begin{aligned}
\mathcal{L}_{\text {soft }}^{\mathcal{Z}_{2}^{\prime} \text { odd }}= & -\left[\sum_{\imath=1}^{2} \tilde{l}_{\imath L}^{c \star}\left(M_{l}^{2}\right)_{\imath 3} \tilde{l}_{3 L}+\sum_{\imath=1}^{2} \tilde{u}_{\imath L}^{c \star}\left(M_{u}^{2}\right)_{\imath 3} \tilde{u}_{3 L}^{c}+\sum_{\imath=2}^{3} \tilde{d}_{\imath L}^{c \star}\left(M_{d}^{2}\right)_{\imath 1} \tilde{d}^{c}{ }_{1 L}\right] \\
& -\left[\sum_{\imath=1}^{3} \sum_{\jmath=2}^{3} A_{\imath \jmath}^{d} H_{1} \tilde{Q}_{\imath L} \tilde{d}_{\jmath L}^{c}+\sum_{\imath=1}^{3} A_{\imath 3}^{u} H_{2} \tilde{Q}_{\imath L} \tilde{u}_{3 L}^{c}+\sum_{\imath=1}^{3} A_{\imath 3}^{l} H_{1} \tilde{L}_{\imath L} \tilde{l}_{3 L}^{c}+\text { h.c. }\right] .
\end{aligned}
$$

It worth remembering the scalar masses $M_{Q}^{2}, M_{u}^{2}, M_{d}^{2}, M_{L}^{2}$, and $M_{l}^{2}$ are in general $3 \times 3$ hermitian matrices in generation space, while $A^{u}, A^{d}$, and $A^{l}$ are general $3 \times 3$ matrices. Allowing all the parameters in eqs. (3.2), (3.3) to be complex, we end up with 124 masses, phases and mixing angles as free parameters of the model.

Constraints from FCNC processes also restrict the form of the soft SUSY breaking trilinear terms $A^{u}, A^{d}$ and $A^{e}$. For example, the data from $K^{0} \bar{K}^{0}$ mixing require the off-diagonal entries of the $A^{d}$ matrix to be small.

Besides, these terms make contributions to fermion masses [11, 12, 17- 21]. The requirement that contributions to the fermion masses to be smaller than the fermion masses themselves put tight constraints to the magnitudes of the $A$-terms.

As we said at section 2 if our $\mathcal{Z}_{2}^{\prime}$ is hold there is no contribution to the EDM. However we have to break this symmetry in order to generate masses to the fermions and we also allow contributions to the EDM. Limits on the imaginary part of the soft SUSY breaking $A$-terms can be obtained from experimental upper limits of electron and neutron EDM [12]

$$
\begin{aligned}
d_{e} & \propto \sqrt{\Im\left(A^{e}\right) v_{1}}<6 \cdot 10^{-4} m_{\tilde{e}}, \\
d_{n} & \propto \sqrt{\Im\left(A^{d}\right) v_{1}}<0,002 m_{\tilde{d}} .
\end{aligned}
$$

Here, we consider the most general scenario which is called in the literature as nonminimal flavor scenario. We follow reference [26] and the sfermions fields are rearranged into the following vector with six component:

$$
\tilde{f}^{T}=\left(\begin{array}{ll}
\tilde{f}_{\imath L} & \tilde{f}_{\imath R}
\end{array}\right),
$$


where each $\tilde{f}_{\imath L}, \tilde{f}_{\imath R}$ is a three component column vector in generation space, ${ }^{4} f=u, d, l$ and $\imath=1,2,3$. Then we can write sfermion mass term of the MSSM Lagrangian in the following way:

$$
\tilde{f}^{\dagger} \mathcal{M}_{\tilde{f}}^{2} \tilde{f}
$$

where $\mathcal{M}_{\tilde{f}}^{2}$ are $6 \times 6$ sfermion mass matrices - one for up-type, one for down-type squarks and one for charged sleptons.

The general squared mass matrix of sfermions can be written as a $2 \times 2$ Hermitian matrix of $3 \times 3$ blocks in the space spanned by the vector of eq. (3.5) [11]

$$
\mathcal{M}_{\tilde{f}}^{2}=\left(\begin{array}{ll}
\mathcal{M}_{\tilde{f}_{\mathrm{L}} \mathrm{L}}^{2} & \mathcal{M}_{\tilde{f}_{\mathrm{LR}}}^{2} \\
\mathcal{M}_{\tilde{f}_{\mathrm{LR}}}^{2 \dagger} & \mathcal{M}_{\tilde{f}_{\mathrm{RR}}}^{2}
\end{array}\right) .
$$

The squared mass matrix of sfermions are diagonalized by pairs of $3 \times 6$ matrices as follows:

$$
\begin{aligned}
& \operatorname{diag}\left(m_{\widetilde{u}_{1}}^{2} \ldots m_{\widetilde{u}_{6}}^{2}\right)=\left(W^{\tilde{u}_{L} \dagger} W^{\tilde{u}_{R} \dagger}\right) \mathcal{M}_{\tilde{u}}^{2}\left(\begin{array}{c}
W^{\tilde{u}_{L}} \\
W^{\tilde{u}_{R}}
\end{array}\right), \\
& \operatorname{diag}\left(m_{\widetilde{d}_{1}}^{2} \ldots m_{\widetilde{d}_{6}}^{2}\right)=\left(W^{\tilde{d}_{L} \dagger} W^{\tilde{d}_{R} \dagger}\right) \mathcal{M}_{\tilde{d}}^{2}\left(\begin{array}{c}
W^{\tilde{d}_{L}} \\
W^{\tilde{d}_{R}}
\end{array}\right), \\
& \operatorname{diag}\left(m_{\tilde{l}_{1}}^{2} \ldots m_{\tilde{l}_{6}}^{2}\right)=\left(W^{\tilde{E}_{L} \dagger} W^{\tilde{E}_{R} \dagger}\right) \mathcal{M}_{\tilde{l}}^{2}\left(\begin{array}{c}
W^{\tilde{E}_{L}} \\
W^{\tilde{E}_{R}}
\end{array}\right) .
\end{aligned}
$$

However, it is common to rotate quarks to their mass eigenstates basis and to rotate squarks in exactly the same way as quarks. This is the so-called Super-CKM (SCKM) basis. It is a suitable basis for the study of flavor violation process since all the unphysical parameters in the Yukawa matrices have already been rotated away, see at ref. 26].

\subsubsection{The squarks}

Here we present the constraints on the elements of squarks mass matrix due to our $\mathcal{Z}_{2}^{\prime}$ symmetry. It is worth recalling the hypothesis of misalignment between the squark and quark mass matrices is present in the most general parameterization of the MSSM and it generates dangerous FCNC effects in conflict with experiment. Specially, the data on $K^{0}-\bar{K}^{0}$ and $D^{0}-\bar{D}^{0}$ mixing impose severe constraints on the mixing involving the $u$-squark and $d$-squark [13].

However, as discussed at the beginning of this section, only particles with the same quantum number can mix with each other. On the other hand, $H_{1,2}$ are even under the $\mathcal{Z}_{2}^{\prime}$ symmetry. Thus $\mathcal{Z}_{2}^{\prime}$ symmetry is maintained in the presence of the spontaneous breaking of gauge symmetry as can be shown by eq. (3.2).

It is useful to stress the parameters $A_{i b}^{d}$ and $A_{i 3}^{u}$ (eq. (3.3)) should be zero because they are not allowed by our $\mathcal{Z}_{2}^{\prime}$ symmetry. This means the third family does not mix with other two families of squarks, then the following matrix elements of eq. (3.7) vanished:

$$
\begin{aligned}
& \left(\mathcal{M}_{\tilde{f}_{\mathrm{LR}}}^{2}\right)_{31}=\left(\mathcal{M}_{\tilde{f}_{\mathrm{LR}}}^{2}\right)_{32}=\left(\mathcal{M}_{\tilde{f}_{\mathrm{LR}}}^{2}\right)_{34}=\left(\mathcal{M}_{\tilde{f}_{\mathrm{LR}}}^{2}\right)_{35}=0, \\
& \left(\mathcal{M}_{\tilde{f}_{\mathrm{LR}}}^{2}\right)_{61}=\left(\mathcal{M}_{\tilde{f}_{\mathrm{LR}}}^{2}\right)_{62}=\left(\mathcal{M}_{\tilde{f}_{\mathrm{LR}}}^{2}\right)_{64}=\left(\mathcal{M}_{\tilde{f}_{\mathrm{LR}}}^{2}\right)_{65}=0,
\end{aligned}
$$

\footnotetext{
${ }^{4}$ We want to emphasize that $\tilde{f}$ is the superpartner of any matter fermion field $f$.
} 
and we obtain the same texture of mass matrix of squark as in reference [27], but only with the requirement of invariance under $\mathcal{Z}_{2}^{\prime}$ :

$$
\mathcal{M}_{\tilde{u}\{\tilde{d}\}}^{2}=\left(\begin{array}{cccccc}
M_{\tilde{L} c\{s\}}^{2} & \left(M_{\tilde{U}\{\tilde{D}\}}^{2}\right)_{\mathrm{LL}} & 0 & m_{c\{s\}} \mathcal{A}_{c\{s\}} & \left(M_{\tilde{U}\{\tilde{D}\}}^{2}\right)_{\mathrm{LR}} & 0 \\
\left(M_{\tilde{U}\{\tilde{D}\}}^{2}\right)_{\mathrm{LL}} & M_{\tilde{L} t\{b\}}^{2} & 0 & \left(M_{\tilde{U}\{\tilde{D}\}}^{2}\right)_{\mathrm{RL}} & m_{t\{b\}} \mathcal{A}_{t\{b\}} & 0 \\
0 & 0 & M_{\tilde{L} u\{d\}}^{2} & 0 & 0 & m_{u\{d\}} \mathcal{A}_{u\{d\}} \\
m_{c\{s\}} \mathcal{A}_{c\{s\}} & \left(M_{\tilde{U}\{\tilde{D}\}}^{2}\right)_{\mathrm{RL}} & 0 & M_{\tilde{R} c\{s\}}^{2} & \left(M_{\tilde{U}\{\tilde{D}\}}^{2}\right)_{\mathrm{RR}} & 0 \\
\left(M_{\tilde{U}\{\tilde{D}\}}^{2}\right)_{\mathrm{LR}} & m_{t\{b\}} \mathcal{A}_{t\{b\}} & 0 & \left(M_{\tilde{U}\{\tilde{D}\}}^{2}\right)_{\mathrm{RR}} & M_{\tilde{R} t\{b\}}^{2} & 0 \\
0 & 0 & m_{u\{d\}} \mathcal{A}_{u,\{d\}} & 0 & 0 & M_{\tilde{R} u\{d\}}^{2}
\end{array}\right),
$$

with

$$
\begin{aligned}
M_{\tilde{L} q}^{2} & =M_{Q, q}^{2}+m_{q}^{2}+\cos 2 \beta\left(T_{q}-Q_{q} s_{W}^{2}\right) M_{Z}^{2}, \\
M_{\tilde{R}\{u, c, t\}}^{2} & =M_{u,\{u, c, t\}}^{2}+m_{u, c, t}^{2}+\cos 2 \beta Q_{t} s_{W}^{2} M_{Z}^{2}, \\
M_{\tilde{R}\{d, s, b\}}^{2} & =M_{d,\{d, s, b\}}^{2}+m_{d, s, b}^{2}+\cos 2 \beta Q_{b} s_{W}^{2} M_{Z}^{2}, \\
\mathcal{A}_{u, c, t} & =\sum_{\imath=1}^{3} A_{3 i, 2 \imath, 1 \imath}^{u, c, t}-\mu \cot \beta, \quad \mathcal{A}_{d, s, b}=\sum_{\imath=1}^{3} A_{3 i, 2 i, 1 \imath}^{d, s, b}-\mu \tan \beta,
\end{aligned}
$$

where $m_{q}, T_{q}, Q_{q}$ are, respectively, the mass, isospin, and electric charge of the quark $q, M_{Z}$ is the mass of $Z$-boson, $s_{W} \equiv \sin \theta_{W}$ and $\theta_{W}$ is the electroweak mixing angle. The masses $m_{u}$ and $m_{d}$ are null, we keep them here only to show that in diagonalization procedure they give rise to the mixing in the third family.

The flavor-changing entries are contained in

$$
\begin{array}{lll}
\left(M_{\widetilde{U}}^{2}\right)_{\mathrm{LL}}=V_{U_{L}} M_{Q}^{2^{*}} V_{U_{L}}^{\dagger}, & \left(M_{\widetilde{U}}^{2}\right)_{\mathrm{RR}}=V_{U_{R}} M_{u}^{2^{*}} V_{U_{R}}^{\dagger}, & \left(M_{\widetilde{U}}^{2}\right)_{\mathrm{LR}}=v_{u}^{*} V_{U_{L}} A_{u}^{*} V_{U_{R}}^{\dagger}, \\
\left(M_{\widetilde{D}}^{2}\right)_{\mathrm{LL}}=V_{D_{L}} M_{Q}^{2^{*}} V_{D_{L}}^{\dagger}, & \left(M_{\widetilde{D}}^{2}\right)_{\mathrm{RR}}=V_{D_{R}} M_{d}^{2^{*}} V_{D_{R}}^{\dagger}, \quad\left(M_{\widetilde{D}}^{2}\right)_{\mathrm{LR}}=v_{d}^{*} V_{D_{L}} A_{d}^{*} V_{D_{R}}^{\dagger}
\end{array}
$$

Eq. (3.14) demonstrates the needs of all four matrices $V_{U, D_{L, R}}$ even though the observed CKM matrix only constraints one combination of them.

Each one of general six by six mass matrix of eq. (3.12) can be split into two matrices: one of order four and the other of order two. The order four matrix corresponds to the mass and mixing of squarks of first and second families while the masses and mixing of squarks of third family constitute the mass matrix of order two. One performs the diagonalization procedure of the matrices in the following way:

(a-) Mixing between the first and second family of the squarks

The four component vectors for up-squark and down-squarks are, respectively, $\left(\tilde{u}_{1 L}, \tilde{u}_{2 L}, \tilde{u}_{1 R}, \tilde{u}_{2 R}\right)$ and $\left(\tilde{d}_{1 L}, \tilde{d}_{2 L} \tilde{d}_{1 R}, \tilde{d}_{2 R}\right)$. Thus the squark squared mass matrices are given by:

$$
\mathcal{M}_{\tilde{u}\{\tilde{d}\}}^{2}=\left(\begin{array}{llll}
M_{\tilde{L}, c\{s\}}^{2} & \left(M_{\tilde{U}\{\tilde{D}\}}^{2}\right)_{\mathrm{LL}} & m_{c\{s\}} \mathcal{A}_{c\{s\}} & \left(M_{\tilde{U}\{\tilde{D}\}}^{2}\right)_{\mathrm{LR}} \\
\left(M_{\tilde{U}\{\tilde{D}\}}^{2}\right)_{\mathrm{LL}} & M_{\tilde{L} t\{b\}}^{2} & \left(M_{\tilde{U}\{\tilde{D}\}}^{2}\right)_{\mathrm{RL}} & m_{t\{b\}} \mathcal{A}_{t\{b\}} \\
\left(M_{\tilde{U}\{\tilde{D}\}}^{2}\right)_{\mathrm{LR}} & \left(M_{\tilde{U}\{\tilde{D}\}}^{2}\right)_{\mathrm{RL}} & M_{\tilde{R} c\{s\}}^{2} & \left(M_{\tilde{U}\{\tilde{D}\}}^{2}\right)_{\mathrm{RR}} \\
\left(M_{\tilde{U}\{\tilde{D}\}}^{2}\right)_{\mathrm{LR}} & m_{t\{b\}} \mathcal{A}_{t\{b\}} & \left(M_{\tilde{U}\{\tilde{D}\}}^{2}\right)_{\mathrm{RR}} & M_{\tilde{R} t\{b\}}^{2}
\end{array}\right) .
$$


In order to diagonalize $\mathcal{M}_{\tilde{u}\{\tilde{d}\}}^{2}$ one requires two rotation $4 \times 4$ matrices: one for the up-squarks $\left(R^{(u)}\right)$ and one for down-squarks $\left(R^{(d)}\right)$. Thus the squark mass eigenstates $\left(\tilde{q}_{\alpha}^{\prime}\right)$ and the interaction squark eigenstates $\left(\tilde{q}_{\alpha}\right)$ are related by,

$$
\tilde{q}_{\alpha}^{\prime}=\sum R_{\alpha \beta}^{(q)} \tilde{q}_{\beta}
$$

where explicitly the matrices reads

$$
\tilde{u}_{\alpha}^{\prime}=\left(\begin{array}{c}
\tilde{c}_{L} \\
\tilde{c}_{R} \\
\tilde{t}_{L} \\
\tilde{t}_{R}
\end{array}\right), \tilde{d}_{\alpha}^{\prime}=\left(\begin{array}{c}
\tilde{s}_{L} \\
\tilde{s}_{R} \\
\tilde{b}_{L} \\
\tilde{b}_{R}
\end{array}\right), \tilde{u}_{\beta}=\left(\begin{array}{c}
\tilde{u}_{1 L} \\
\tilde{u}_{2 L} \\
\tilde{u}_{1 R} \\
\tilde{u}_{2 R}
\end{array}\right), \tilde{d}_{\beta}=\left(\begin{array}{c}
\tilde{d}_{1 L} \\
\tilde{d}_{2 L} \\
\tilde{d}_{1 R} \\
\tilde{d}_{2 R}
\end{array}\right) .
$$

One obtains the squark mass eigenvalues and eigenstates after the diagonalization procedure as indicated in ref. [28].

(b-) $u$ and $d$-squarks

In the symmetric case under $\mathcal{Z}_{2}^{\prime}$ the mass matrix is trivially diagonal and $\tilde{q}_{3 L}$ does not mix with $\tilde{q}_{3 R}$, as a consequence the contribution of the squark sector to the EDM is null.

The interesting case comes from the soft breaking terms of eq. (3.3). For the third generation these terms are given by

$M_{Q, 3}^{2} \tilde{u}_{3 L}^{\star} \tilde{u}_{3 L}+M_{u, u}^{2} \tilde{u}_{3 R}^{\star} \tilde{u}_{3 R}+A_{33}^{u} \tilde{u}_{3 L} \tilde{u}_{3 R} v_{2}+M_{Q, 3}^{2} \tilde{d}_{3 L}^{\star} \tilde{d}_{3 L}+M_{d, d}^{2} \tilde{d}_{3 R}^{\star} \tilde{d}_{3 R}+A_{33}^{d} \tilde{d}_{3 L} \tilde{d}_{3 R} v_{1}$

they give the mixing between left-right part of the u-squark and d-squark sector. This mixing has two important consequences:

The first one is the mass of the squarks of the third family. The off diagonal entries are proportional to the mass of quarks as shown below,

$$
\mathcal{M}_{\tilde{q}}^{2}=\left(\begin{array}{cc}
m_{\tilde{q}_{L}}^{2} & a_{q} m_{q} \\
a_{q} m_{q} & m_{\tilde{q}_{R}}^{2}
\end{array}\right)=\left(\mathcal{R}^{\tilde{q}}\right)\left(\begin{array}{cc}
m_{\tilde{q}_{1}}^{2} & 0 \\
0 & m_{\tilde{q}_{2}}^{2}
\end{array}\right) \mathcal{R}^{\tilde{q}}
$$

where $\tilde{q}=\tilde{u}, \tilde{d}$. The weak eigenstates $\tilde{q}_{L}$ and $\tilde{q}_{R}$ are thus related to their mass eigenstates $\tilde{q}_{1}$ and $\tilde{q}_{2}$ by

$$
\left(\begin{array}{c}
\tilde{q}_{1} \\
\tilde{q}_{2}
\end{array}\right)=\mathcal{R}^{\tilde{q}}\left(\begin{array}{c}
\tilde{q}_{3 L} \\
\tilde{q}_{3 R}
\end{array}\right), \quad \mathcal{R}^{\tilde{q}}=\left(\begin{array}{r}
\cos \theta_{\tilde{q}} \sin \theta_{\tilde{q}} \\
-\sin \theta_{\tilde{q}} \cos \theta_{\tilde{q}}
\end{array}\right)
$$

with $\theta_{\tilde{q}}$ the squark mixing angle. The mass eigenvalues are given by

$$
m_{\tilde{q}_{1,2}}^{2}=\frac{1}{2}\left(m_{\tilde{q}_{L}}^{2}+m_{\tilde{q}_{R}}^{2} \mp \sqrt{\left(m_{\tilde{q}_{L}}^{2}-m_{\tilde{q}_{R}}^{2}\right)^{2}+4 a_{q}^{2} m_{q}^{2}}\right) .
$$

By convention, we choose $\tilde{q}_{1}$ to be the lightest mass eigenstate. Note that $m_{\tilde{q}_{1}} \leq$ $m_{\tilde{q}_{L, R}} \leq m_{\tilde{q}_{2}}$. For the mixing angle $\theta_{\tilde{q}}$ we require $0 \leq \theta_{\tilde{q}}<\pi$. Thus, we have

$$
\cos \theta_{\tilde{q}}=\frac{-a_{q} m_{q}}{\sqrt{\left(m_{\tilde{q}_{L}}^{2}-m_{\tilde{q}_{1}}^{2}\right)^{2}+a_{q}^{2} m_{q}^{2}}}, \quad \sin \theta_{\tilde{q}}=\frac{m_{\tilde{q}_{L}}^{2}-m_{\tilde{q}_{1}}^{2}}{\sqrt{\left(m_{\tilde{q}_{L}}^{2}-m_{\tilde{q}_{1}}^{2}\right)^{2}+a_{q}^{2} m_{q}^{2}}}
$$

This mixing is important because it generates contributions to the EDM. 


\subsubsection{The masses of selectrons}

The procedure is the same as in the case of squarks. The mixing in the selectron sector comes from the following $\mathcal{Z}_{2}^{\prime}$-odd terms,

$$
M_{L, 3}^{2} \tilde{l}_{3 L}^{\star} \tilde{l}_{3 L}+M_{l, l}^{2} \tilde{l}_{3 R}^{\star} \tilde{l}_{3 R}+A_{33}^{l} \tilde{l}_{3 L} \tilde{l}_{3 R} v_{1}
$$

The relations among mass eigenstates and interaction eigenstates of selectron are,

$$
\left(\begin{array}{c}
\tilde{e}_{1} \\
\tilde{e}_{2}
\end{array}\right)=\mathcal{R}^{\tilde{e}}\left(\begin{array}{c}
\tilde{l}_{3 L} \\
\tilde{l}_{3 R}
\end{array}\right), \quad \mathcal{R}^{\tilde{e}}=\left(\begin{array}{c}
\cos \theta_{\tilde{e}} \sin \theta_{\tilde{e}} \\
-\sin \theta_{\tilde{e}} \cos \theta_{\tilde{e}}
\end{array}\right)
$$

with $\theta_{\tilde{e}}$ the selectron mixing angle. The mass eigenvalues are the same as in the case of third family of squarks, therefore their masses are given by the eq. (3.21) but with label $q$ instead of $e$.

\subsection{The masses of gluinos}

It is well known gluinos are the supersymmetric partners of the gluons. Therefore gluinos are the color octet fermions in the model. On other hand, as the $\mathrm{SU}(3)_{c}$ group is unbroken gluinos can not mix with any others particles in the model, then they are already mass eigenstates.

Their mass, from eq. (3.2), can be written as

$$
\mathcal{L}_{\text {mass }}^{\text {gluino }}=\frac{m_{\tilde{g}}}{2} \overline{\tilde{g}} \tilde{g}
$$

so that its mass at tree level is $m_{\tilde{g}}=\left|M_{3}\right|$, where

$$
\tilde{g}^{a}=\left(\begin{array}{c}
-\imath \lambda_{C}^{a} \\
\imath \overline{\lambda_{C}^{a}}
\end{array}\right), \quad a=1, \ldots, 8,
$$

is the Majorana four-spinor defining the physical gluinos states.

\section{The mechanism of mass generation}

Once $\mathcal{Z}_{2}^{\prime}$ symmetry is softly broken the fermions are allowed to interact with their respective superpartners and gluinos (see at appendix B). However, the third family is already disconnected from other two families and we show that the removal of alignment assumption only changes the content of strange quark mass.

\subsection{Light fermion masses}

The $u$-quark can only interact with $u$-squark (defined at eq. (3.20)). However, squarks can couple with gluino and also with bino, the supersymmetric partner of the gauge boson of U(1). First we want to compare their contribution to the 1-loop mass diagram which generates mass to the $u$ quark. 


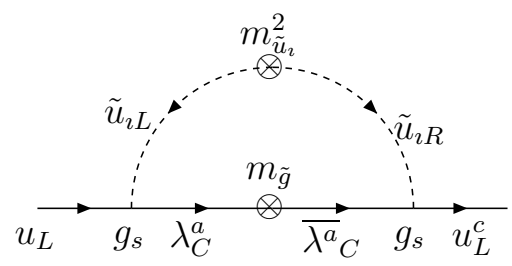

Figure 1: The diagram which gives mass to quark $u \mathrm{l}, \lambda_{C}^{a}$ is the gluino while $\tilde{u}_{i}, i=1,2$, is the u-squark.

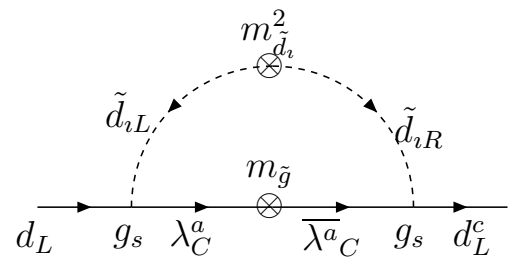

Figure 2: The diagram which gives mass to quark $d, \lambda_{C}^{a}$ is the gluino while $\tilde{d}_{i}, i=1,2$, is the d-squark.

In order to estimate their contribution, it is useful to use the Supersymmetry Parameter Analysis Convention (SPA). Wchich is based on a consistent set of conventions and input parameters [29-31, given at appendix Q. In all the scenarios is easy to see that

$$
g_{s}^{2} m_{\tilde{g}} \gg g^{\prime 2} m^{\prime}
$$

keep this in mind one can neglect the contribution of the bino.

The interaction between the squarks-gluino-quarks is given by eq. (B.3). In figure 1 we depict the loop diagram contribution for the mass of $u$ - quark which gives rise to the following expression as a function of loop integrals ${ }^{5}$ (see at eq. (D.1)):

$$
M_{u}=g_{s}^{2} m_{\tilde{g}} \sin \left(2 \theta_{\tilde{u}}\right) \sum_{\imath=1}^{2} B_{0}\left(m_{\tilde{u}_{\imath}}, m_{\tilde{g}}\right)
$$

Analogously we obtain for the mass of $d$-quark, see figure 2 , the following expression ${ }^{6}$

$$
M_{d}=g_{s}^{2} m_{\tilde{g}} \sin \left(2 \theta_{\tilde{d}}\right) \sum_{\imath=1}^{2} B_{0}\left(m_{\tilde{d}_{\imath}}, m_{\tilde{g}}\right) .
$$

These expressions (4.2), (4.3) agree with the results presented in refs. [17-21].

Likewise the quark case, selectron (eq. (3.24)) interacts with electron (eq. (B.6)) and this interaction is the source for the leading contribution depicted in figure 3. We obtain the following expression for the electron mass

$$
M_{e}=g^{\prime 2} \sin \left(2 \theta_{\tilde{e}}\right) m^{\prime} \sum_{\imath=1}^{2} B_{0}\left(m_{\tilde{e}_{\imath}}, m^{\prime}\right) .
$$

\footnotetext{
${ }^{5} m_{\tilde{u}}$ and $m_{\tilde{g}}$ are the masses of the u-squark and gluinos respectively.

${ }^{6} m_{\tilde{d}}$ is the d-squark mass.
} 


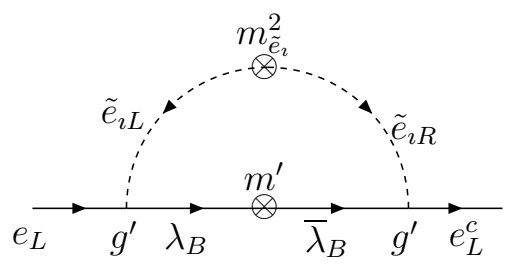

Figure 3: The diagram which gives mass to electron, $\lambda_{B}$ is the bino while $\tilde{e}_{i}, i=1,2$, is the selectron.
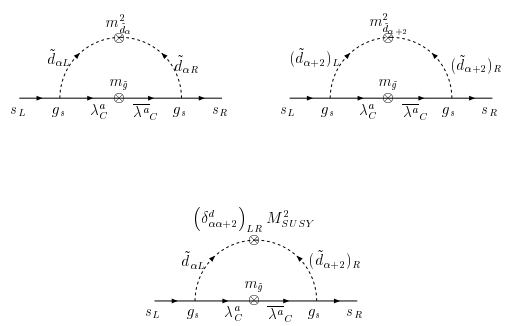

Figure 4: The diagram which gives mass to quark $s, \lambda_{C}^{a}$ is the gluino while $\tilde{s}_{i}$ and $\tilde{b}_{i}, i=1,2$, are the squarks s-squark and sbottom, respectively.

Same as one finds in our first work, the light fermions can couple only with their respective supersymmetric partners. In contrast, now the strange quark can couple with $s$ and $b$-squark, defined at eq. (3.16). This is a source of flavor non-diagonal sfermion mass matrix.

We define the dimensionless flavor-changing parameters $\left(\delta_{\imath \jmath}^{u, d}\right)_{\mathrm{AB}}(A, B=L, R)$ from the flavor off-diagonal elements of the squark mass matrices ( eq. (3.12)), in the following way: first, we set all diagonal entries $M_{Q, q}^{2}$ and $M_{u(d), q}^{2}$ to be equal to the common value $M_{\mathrm{SUSY}}^{2}$, then we normalize the off-diagonal elements to $M_{\mathrm{SUSY}}^{2}$ [13, 26- 28],

$$
\left(\delta_{\imath \jmath}^{d(u)}\right)_{\mathrm{AB}}=\frac{\left(M_{\tilde{U}(\tilde{D})}^{2}\right)_{\mathrm{AB}}^{\imath \jmath}}{M_{\mathrm{SUSY}}^{2}}, \quad(\imath \neq \jmath, \quad \imath, \jmath=1,2 \quad A, B=L, R) .
$$

Due this fact the leading contribution to the mass of $s$-quark is shown in figure 4 . Taking into account eq. (B.4) one obtains the following expression

$$
\begin{aligned}
M_{s}=2 g_{s}^{2} m_{\tilde{g}} \sum_{\alpha=1}^{2}[ & R_{1 \alpha}^{(d)} R_{2 \alpha}^{(d)} B_{0}\left(m_{\tilde{d}_{\alpha}}, m_{\tilde{g}}\right)+R_{1 \alpha+2}^{(d)} R_{2 \alpha+2}^{(d)} B_{0}\left(m_{\tilde{d}_{\alpha+2}}, m_{\tilde{g}}\right) \\
& \left.+R_{1 \alpha}^{(d)} R_{2 \alpha+2}^{(d)}\left(\delta_{\alpha \alpha+2}^{d}\right)_{\mathrm{LR}} M_{\mathrm{SUSY}}^{2} I\left(m_{\tilde{d}_{\alpha}}, m_{\tilde{d}_{\alpha+2}}, m_{\tilde{g}}\right)\right] .
\end{aligned}
$$

It worth noting that the content of mass of $s$ quark is very different from the content of others two light quarks, eqs. (4.3), (4.6), and we are able to make the strange quark heavier than non-strange quarks.

In fact, even if we consider all the squarks are degenerate in mass the strange quark still is heavier than non-strange quarks:

$$
m_{s}>4 m_{d}
$$


This relation is in agreement with recent experimental data [32]

$$
17 \leq \frac{m_{s}}{m_{d}} \geq 22
$$

\subsection{Final expressions}

As it is clear in eqs. (4.2), (4.3), (4.4) we have to perform only one integral. From eq. (D.6), we can rewrite the light fermion mass expressions as follow:

$$
\begin{aligned}
& M_{u}=\frac{g_{s}^{2} m_{\tilde{g}} \sin \left(2 \theta_{\tilde{u}}\right)}{16 \pi^{4}} \sum_{\imath=1}^{2} \frac{m_{\tilde{u}_{\imath}}^{2}}{\left(m_{\tilde{u}_{\imath}}^{2}-m_{\tilde{g}}^{2}\right)} \ln \left(\frac{m_{\tilde{u}_{\imath}}^{2}}{m_{\tilde{g}}^{2}}\right), \\
& M_{d}=\frac{g_{s}^{2} m_{\tilde{g}} \sin \left(2 \theta_{\tilde{d}}\right)}{16 \pi^{4}} \sum_{\imath=1}^{2} \frac{m_{\tilde{d}_{\imath}}^{2}}{\left(m_{\tilde{d}_{\imath}}^{2}-m_{\tilde{g}}^{2}\right)} \ln \left(\frac{m_{\tilde{d}_{\imath}}^{2}}{m_{\tilde{g}}^{2}}\right), \\
& M_{e}=\frac{g^{\prime 2} m^{\prime} \sin \left(2 \theta_{\tilde{e}}\right)}{16 \pi^{4}} \sum_{\imath=1}^{2} \frac{m_{\tilde{e}_{\imath}}^{2}}{\left(m_{\tilde{e}_{\imath}}^{2}-m^{\prime 2}\right)} \ln \left(\frac{m_{\tilde{e}_{\imath}}^{2}}{m^{\prime 2}}\right) .
\end{aligned}
$$

These results agree with literature [18, 21, 33].

From the scenarios SPA, see appendix $\mathbb{Q}$, the expression

$$
\frac{m_{\tilde{g}}^{2}}{\left(m_{\tilde{g}}^{2}-m_{\tilde{q}_{2}}^{2}\right)} \ln \left(\frac{m_{\tilde{g}}^{2}}{m_{\tilde{q}_{2}}^{2}}\right)
$$

has positive values. We can also see that $m_{\tilde{e}_{2}}>m^{\prime}$ and therefore we can use the equation above in order to reproduce the mass pattern of these fermions.

The expression for the mass of $s$ quark has a more complicated integral to be solved, see figure 4 and eq. (4.6) turns into the following:

$$
\begin{gathered}
M_{s}=\frac{g_{s}^{2} m_{\tilde{g}}}{16 \pi^{4}} \sum_{\alpha=1}^{2}\left\{R_{1 \alpha}^{(d)} R_{2 \alpha}^{(d)} \frac{m_{\tilde{g}}^{2}}{\left(m_{\tilde{g}}^{2}-m_{\tilde{d}_{\alpha}}^{2}\right)} \ln \left(\frac{m_{\tilde{g}}^{2}}{m_{\tilde{d}_{\alpha}}^{2}}\right)+R_{1 \alpha+2}^{(d)} R_{2 \alpha+2}^{(d)} \frac{m_{\tilde{g}}^{2}}{\left(m_{\tilde{g}}^{2}-m_{\tilde{d}_{\alpha+2}}^{2}\right)} \ln \left(\frac{m_{\tilde{g}}^{2}}{m_{\tilde{d}_{\alpha+2}}^{2}}\right)\right. \\
+\frac{R_{1 \alpha}^{(d)} R_{2 \alpha+2}^{(d)}}{\left(m_{\tilde{d}_{\alpha}}^{2}-m_{\tilde{d}_{\alpha+2}}^{2}\right)\left(m_{\tilde{g}}^{2}-m_{\tilde{d}_{\alpha}}^{2}\right)\left(m_{\tilde{d}_{\alpha+2}}^{2}-m_{\tilde{g}}^{2}\right)}\left(\delta_{\alpha \alpha+2}^{d}\right)_{\mathrm{LR}} M_{\mathrm{SUSY}}^{2}\left[m_{\tilde{d}_{\alpha}}^{2} m_{\tilde{d}_{\alpha+2}}^{2} \ln \left(\frac{m_{\tilde{d}_{\alpha}}^{2}}{m_{\tilde{d}_{\alpha+2}}^{2}}\right)\right. \\
\left.\left.+m_{\tilde{d}_{\alpha}}^{2} m_{\tilde{g}}^{2} \ln \left(\frac{m_{\tilde{g}}^{2}}{m_{\tilde{d}_{\alpha}}^{2}}\right)+m_{\tilde{d}_{\alpha+2}}^{2} m_{\tilde{g}}^{2} \ln \left(\frac{m_{\tilde{d}_{\alpha+2}}^{2}}{m_{\tilde{g}}^{2}}\right)\right]\right\} .
\end{gathered}
$$

It is important to emphasize that the first two contribution to the mass of this quark are the same as those in the mass expressions of $u$ and $d$ quarks. The third contribution came from the flavor non-diagonal sfermion mass matrix contribution. As a result of small mixing, the mass eigenstates are approximately equal to the flavor eigenstates and hence approximate flavor eigenstates are propagating in the loop (squarks $\tilde{d}_{1}$ and $\tilde{d}_{3}$ or $\tilde{d}_{2}$ and $\left.\tilde{d}_{4}\right)$, this mixing couples squark of different flavors $\left(\delta_{13}^{d}\right.$ and $\left.\delta_{24}^{d}\right)$.

\section{Conclusions}

We showed that the extension of $\mathcal{Z}_{2}^{\prime}$ symmetry to the squarks sector provide us with a natural mechanism for explaining the chiral mass hierarchy pattern and also the mass 


\begin{tabular}{|c|cc|cc|}
\hline Superfield & Usual Particle & Spin & Superpartner & Spin \\
\hline$\hat{V}^{\prime}(\mathrm{U}(1))$ & $V_{m}$ & 1 & $\lambda_{B}$ & $\frac{1}{2}$ \\
$\hat{V}^{\imath}(\mathrm{SU}(2))$ & $V_{m}^{\imath}$ & 1 & $\lambda_{A}^{\imath}$ & $\frac{1}{2}$ \\
$\hat{V}_{c}^{a}(\mathrm{SU}(3))$ & $G_{m}^{a}$ & 1 & $\tilde{g}^{a}$ & $\frac{1}{2}$ \\
\hline$\hat{Q}_{\imath} \sim(\mathbf{3}, \mathbf{2}, 1 / 3)$ & $\left(u_{\imath}, d_{\imath}\right)_{L}$ & $\frac{1}{2}$ & $\left(\tilde{u}_{\imath L}, \tilde{d}_{\imath L}\right)$ & 0 \\
$\hat{u}_{\imath}^{c} \sim\left(\mathbf{3}^{*}, \mathbf{1},-4 / 3\right)$ & $\bar{u}_{\imath L}^{c}$ & $\frac{1}{2}$ & $\tilde{u}_{\imath L}^{c}$ & 0 \\
$\left.\hat{d}_{\imath}^{c} \sim\left(\mathbf{3}^{*}, \mathbf{1}, 2 / 3\right)\right)$ & $\bar{d}_{\imath L}^{c}$ & $\frac{1}{2}$ & $\tilde{d}_{\imath L}^{c}$ & 0 \\
\hline$\hat{L}_{\imath} \sim(\mathbf{1}, \mathbf{2},-1)$ & $\left(\nu_{\imath}, l_{\imath}\right)_{L}$ & $\frac{1}{2}$ & $\left(\tilde{\nu}_{\imath L}, \tilde{l}_{\imath L}\right)$ & 0 \\
$\hat{l}_{l}^{c} \sim(\mathbf{1}, \mathbf{1}, 2)$ & $\bar{l}_{\imath L}^{c}$ & $\frac{1}{2}$ & $\tilde{l}_{\imath L}^{c}$ & 0 \\
\hline$\hat{H}_{1} \sim(\mathbf{1}, \mathbf{2},-1)$ & $\left(H_{1}^{0}, H_{1}^{-}\right)$ & 0 & $\left(\tilde{H}_{1}^{0}, \tilde{H}_{1}^{-}\right)$ & $\frac{1}{2}$ \\
$\hat{H}_{2} \sim(\mathbf{1}, \mathbf{2}, 1)$ & $\left(H_{2}^{+}, H_{2}^{0}\right)$ & 0 & $\left(\tilde{H}_{2}^{+}, \tilde{H}_{2}^{0}\right)$ & $\frac{1}{2}$ \\
\hline
\end{tabular}

Table 1: Particle content of MSSM.

gap between strange and non-strange quarks. The FCNC problems are under control under $R$-parity invariance requirements and the breaking of $\mathcal{Z}_{2}^{\prime}$ symmetry only by SUSY soft terms. There is no need of further assumptions as the alignment between quark and squark sectors or setting null entries for a particular mass matrix elements of squarks. The requirement of non-invariance under $\mathcal{Z}_{2}^{\prime}$ symmetry for the third family of quarks (squarks) disconnects this family from the other two families of quarks (squarks). In the quark sector this disconnection gives rise to the Chiral symmetry breaking only in the heavy quarks sector $(c, t$ and $b)$ while the light quarks remain massless. For squarks sector the family disconnection gives rise to a particular texture for the mass matrix consistent with experimental bonds. Once $\mathcal{Z}_{2}^{\prime}$ is softly broken the light fermions can interact with sfermions and gauginos and they acquire masses by means of radiative mechanism. Thus we can give a reasonable explanation of the mass gap between $s$ quark and non strange quarks, even in the case of all squarks are degenerate in mass ${ }^{7}$ at low energy. It is due to the fact that the $s$ quark can couple with two families of squarks while the $u$ and $d$ quarks can couple only with one family.

\section{Acknowledgments}

M.C. Rodriguez is supported by Conselho Nacional de Ciê ncia e Tecnologia (CNPq) under the contract number 309564/2006-9. C.M. Maekawa was partially supported by FAPERGS PROADE-2 under contract number 02/1266-6.

\section{A. Notation}

In this first appendix we show our notation to the Minimal Supersymmetric Model (MSSM).

\section{A.1 The fields of MSSM}

The particle content of the model is given at table (1). The families index for fermions are

\footnotetext{
${ }^{7}$ We get naturally $m_{s}>4 \times m_{d}$
} 
$\imath, \jmath=1,2,3$. The parentheses in the first column are the transformation properties under the respective representation of $\left(\mathrm{SU}(3)_{C}, \mathrm{SU}(2)_{L}, \mathrm{U}(1)_{Y}\right)$.

\section{B. Interaction of fermion-sfermion-gauginos}

We present the interactions of sfermions with gauginos.

The interaction between Quark-Squarks-Gluino are given by

$$
\mathcal{L}_{q \tilde{q} \tilde{g}}=-\imath \sqrt{2}\left[\tilde{Q} T^{a} \bar{Q} \bar{\lambda}_{C}-\overline{\tilde{Q}} T^{a} Q \lambda_{C}^{a}+\tilde{u}^{c} T^{a} \overline{u^{c}} \lambda^{a}{ }_{C}-\overline{\tilde{u}^{c}} T^{a} u^{c} \lambda_{C}^{a}+\tilde{d}^{c} T^{a} \overline{d^{c} \lambda^{a}}{ }_{C}-\overline{\tilde{d}^{c}} T^{a} d^{c} \lambda_{C}^{a}\right]
$$

in the basis of mass eigenstates we rewrite it as follow:

$$
\mathcal{L}_{q \tilde{q} \tilde{g}}=-\sqrt{2} \sum_{q=u, d} \bar{q}_{\imath}\left[U_{\jmath \imath}^{q_{L} *} W_{\jmath s}^{\tilde{q}} P_{R}-U_{\jmath R^{*}}^{q_{R}} W_{\jmath+3 s}^{\tilde{q}} P_{L}\right] T^{a} \tilde{g}^{a} \tilde{q}_{s}+\text { h.c. },
$$

where $T^{a}$ are the $\mathrm{SU}(3)_{c}$ generators, $P_{L, R} \equiv\left(1 \mp \gamma_{5}\right) / 2, i, j, s=1,2$ are generation indices. In the gluino interaction, the flavor changing effects from soft broken terms $M_{\tilde{Q}}^{2}, M_{\tilde{U}}^{2}$ and $A_{u}$ on the observable are introduced through the matrix $W^{\tilde{q}}$.

To $u$-squark and $d$-squark we can write

$$
\mathcal{L}_{q \tilde{q} \tilde{g}}=-\sqrt{2} \sum_{q=u, d} g_{s} T_{r s}^{a}\left[\bar{q}_{r}\left(\mathcal{R}_{\imath 1}^{\tilde{q}} P_{R}-\mathcal{R}_{\imath 2}^{\tilde{q}} P_{L}\right) \tilde{g}^{a} \tilde{q}_{\imath, s}+h c\right]
$$

The previously introduced intergeneration mixing effects in the squark sector give rise to strong Flavor Changing effects in processes with neutral currents through the quarksquark-gluino interaction Lagrangian, which can now be written in the squark mass eigenstates basis as,

$$
\begin{array}{r}
\mathcal{L}_{q \tilde{q} \tilde{g}}=-\sqrt{2} g_{s} T_{\alpha u}^{a}\left(R_{1 \alpha}^{(u) *} \overline{\tilde{g}}^{a} \tilde{u}_{\alpha}^{*} c_{\mathrm{uL}}+R_{1 \alpha}^{(d) *} \overline{\tilde{g}}^{a} \tilde{d}_{\alpha}^{*} s_{\mathrm{uL}}-R_{2 \alpha}^{(u) *} \overline{\tilde{g}}^{a} \tilde{u}_{\alpha}^{*} c_{\mathrm{uR}}-R_{2 \alpha}^{(d) *} \overline{\tilde{g}}^{a} \tilde{d}_{\alpha}^{*} s_{\mathrm{uR}}\right. \\
\left.+R_{3 \alpha}^{(u) *} \overline{\tilde{g}}^{a} \tilde{u}_{\alpha}^{*} t_{\mathrm{uL}}+R_{3 \alpha}^{(d) *} \overline{\tilde{g}}^{a} \tilde{d}_{\alpha}^{*} b_{\mathrm{uL}}-R_{4 \alpha}^{(u) *} \overline{\tilde{g}}^{a} \tilde{u}_{\alpha}^{*} t_{\mathrm{uR}}-R_{4 \alpha}^{(d) *} \overline{\tilde{g}}^{a} \tilde{d}_{\alpha}^{*} b_{\mathrm{uR}}\right)+h . c
\end{array}
$$

with $\alpha=1,2,3,4$. For simplicity, we will omit the color indices from now on.

On the other hand, the Feynmann rules between Lepton-Slepton-Gaugino is computed from

$$
\begin{aligned}
\mathcal{L}_{\tilde{\chi} \tilde{l} l}= & -\imath \sqrt{2} g T^{\imath}\left(\tilde{L} \bar{L} \bar{\lambda}_{A}^{\imath}-\overline{\tilde{L}} L \lambda_{A}^{\imath}\right)-\frac{\imath g^{\prime}}{\sqrt{2}}(-1)\left(\tilde{L} \bar{L} \bar{\lambda}_{B}-\overline{\tilde{L}} L \lambda_{B}\right) \\
& -\frac{\imath g^{\prime}}{\sqrt{2}} 2\left(\tilde{l}^{c} \overline{l^{c}} \bar{\lambda}_{B}-\overline{\tilde{l}^{c}} l^{c} \lambda_{B}\right),
\end{aligned}
$$

in terms of masses eigenstates we get the following interaction to Lepton-Slepton-Neutralino

$$
\mathcal{L}_{l i \tilde{\chi}^{0}}=\left(\overline{\tilde{\chi}^{0}}\right)_{l}\left(G_{\imath s l}^{e_{L}} P_{L}+G_{\imath s l}^{e_{R}} P_{R}\right) \tilde{e}_{s}^{\dagger} e_{\imath}+\text { h.c. },
$$

where

$$
\begin{aligned}
& G_{\imath s l}^{e_{L}}=G_{l}^{e_{L}} W_{\imath s}^{\tilde{e} \star}-\frac{g}{\sqrt{2} M_{W} \cos \beta} m_{e_{\imath}} Z_{l 3}^{\star} W_{(\imath+3) s}^{\tilde{e} \star}, \\
& G_{\imath s l}^{e_{R}}=G_{l}^{e_{R}} W_{(\imath+3) s}^{\tilde{e} \star}-\frac{g}{\sqrt{2} M_{W} \cos \beta} m_{e_{\imath}} Z_{l 3} W_{\imath s}^{\tilde{e} \star} .
\end{aligned}
$$




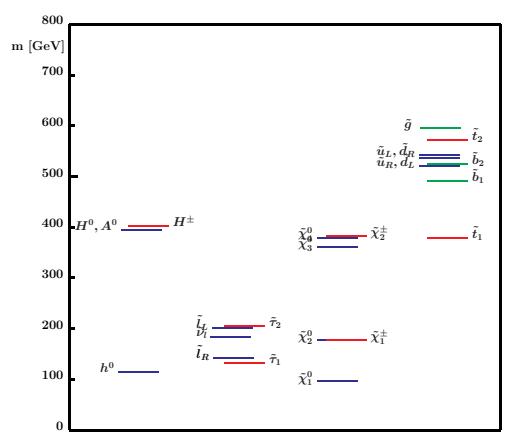

Figure 5: The SUSY particle spectra for the benchmark points corresponding to SPS 1a [30].

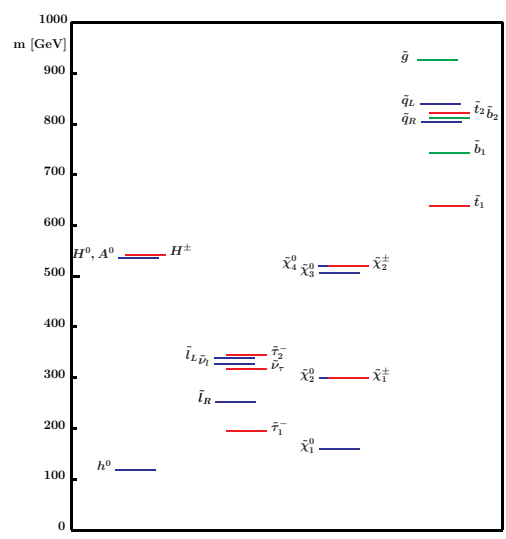

Figure 6: The SUSY particle spectra for the benchmark points corresponding to SPS 1b [30].

\section{SPA convention}

The Supersymmetry Parameter Analysis project (SPA) is a comparative study of supersymmetric particle spectra calculated for various SUSY scenarios [29-31]. The definition of several scenarios are given at [30, 29].

The figures 5, 6, 7, 8 show the particle spectra corresponding to SPS1a, SPS1b and SPS3 [30], where the gluinos are the heavy particles. Also in the scenarios SPS5, SPS6, SPS7 and SPS9 the gluinos are also the heaviest particles. Thus, it is simple to show that eq. (4.10) has positive values. We can also see that $m_{\tilde{e}_{2}}>m^{\prime}$ and therefore we can use the equation above in order to reproduce the mass pattern of these fermions.

At the scenarios SPS2 and SPS8 the gluino are the lightest colored particle and in the last scenario SPS4 we know that $m_{\tilde{g}}<m_{\tilde{q}_{L}, \tilde{q}_{R}}$ then in both case eq. (4.10) still have positive values.

\section{Feynman integration}

We define the following two point function in following way

$$
B_{0}\left(p_{1}^{2}, m_{1}^{2}, m_{2}^{2}\right)=-16 \pi \imath \int \frac{d^{4} k}{(2 \pi)^{4}} \frac{1}{\left[\left(k+p_{1}\right)^{2}-m_{1}^{2}\right]\left[k^{2}-m_{2}^{2}\right]},
$$




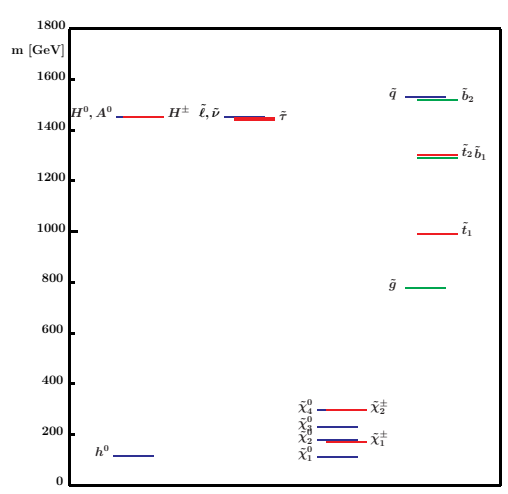

Figure 7: The SUSY particle spectra for the benchmark points corresponding to SPS 2 30.

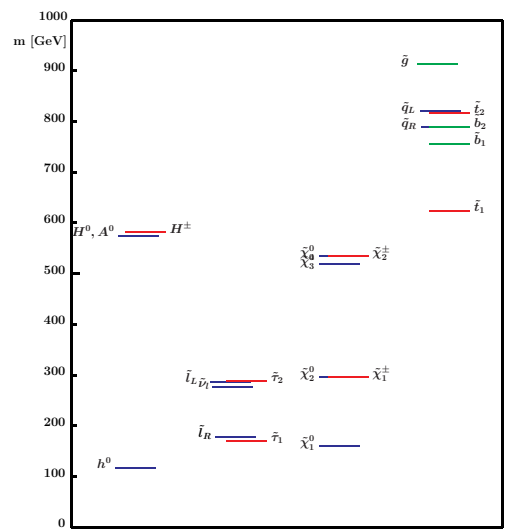

Figure 8: The SUSY particle spectra for the benchmark points corresponding to SPS 3 30.

when the external momentum of the particle is zero, we use the following convention $B_{0}\left(0, m_{1}^{2}, m_{2}^{2}\right) \equiv B_{0}\left(m_{1}, m_{2}\right)$. Perform the integral we get the following result [34]

$$
B_{0}\left(m_{1}, m_{2}\right)=1+\ln \left(\frac{Q^{2}}{m_{2}^{2}}\right)+\frac{m_{1}^{2}}{m_{2}^{2}-m_{1}^{2}} \ln \left(\frac{m_{2}^{2}}{m_{1}^{2}}\right),
$$

where $Q$ is the renormalization scale. After absorb the divergent terms we can rewrite our result as

$$
B_{0}\left(m_{1}, m_{2}\right)=\frac{m_{1}^{2}}{m_{2}^{2}-m_{1}^{2}} \ln \left(\frac{m_{2}^{2}}{m_{1}^{2}}\right)=\frac{m_{1}^{2}}{m_{1}^{2}-m_{2}^{2}} \ln \left(\frac{m_{1}^{2}}{m_{2}^{2}}\right) .
$$

It reproduces the very known results presented at [17- 21, 33, 35].

Now, we are going to analyze the third integral on eq. (4.6). It is an integral of the following form

$$
I\left(m_{1}, m_{2}, m_{3}\right)=\int \frac{d^{4} p}{(2 \pi)^{4}} \frac{1}{\left(p^{2}-m_{1}^{2}\right)} \frac{1}{\left(p^{2}-m_{2}^{2}\right)} \frac{1}{\left(p^{2}-m_{3}^{2}\right)},
$$

One uses eq. (D.1) in order to rewrite eq. (D.4) in the following way

$$
I\left(m_{1}, m_{2}, m_{3}\right)=\frac{1}{m_{1}^{2}-m_{2}^{2}}\left(B_{0}\left(m_{1}, m_{3}\right)-B_{0}\left(m_{2}, m_{3}\right)\right) .
$$


We can also apply eq. (D.3) to show that

$$
\begin{aligned}
I\left(m_{1}, m_{2}, m_{3}\right)=\frac{1}{\left(m_{1}^{2}-m_{2}^{2}\right)\left(m_{1}^{2}-m_{3}^{2}\right)\left(m_{2}^{2}-m_{3}^{2}\right)} & {\left[m_{1}^{2} m_{2}^{2} \ln \left(\frac{m_{1}^{2}}{m_{2}^{2}}\right)\right.} \\
& \left.+m_{1}^{2} m_{3}^{2} \ln \left(\frac{m_{3}^{2}}{m_{1}^{2}}\right)+m_{2}^{2} m_{3}^{2} \ln \left(\frac{m_{2}^{2}}{m_{3}^{2}}\right)\right] .
\end{aligned}
$$

These results are the same as the function $F(x, y, z)$ of refs. [19, 20].

\section{References}

[1] A.D. Sakarov, Violation of CP invariance, $C$ asymmetry, and baryon asymmetry of the universe, Pisma Zh. Eksp. Teor. Fiz. 5 (1967) 32 JETP Lett. 5 (1967) 24, Sov. Phys. Usp. 34 (1991) 392] Usp. Fiz. Nauk. 161 (1991) 61];

I.I. Bigi and A.I. Sanda, CP Violation, Cambridge University Press, Cambridge U.K. (2000).

[2] S. Weinberg, Baryon and lepton nonconserving processes, Phys. Rev. Lett. 43 (1979) 1566.

[3] M. Diwan et al., Proposal for an experimental program in neutrino physics and proton decay in the homestake laboratory, hep-ex/0608023.

[4] N.F. Ramsey, Electric dipole moments of elementary particles, Rept. Prog. Phys. 45 (1982) 95 .

[5] A. Gal, Limits on $n \bar{n}$ oscillations from nuclear stability, Phys. Rev. C 61 (2000) 028201 hep-ph/9907334.

[6] S.L. Glashow, Partial symmetries of weak interactions, Nucl. Phys. 22 (1961) 579,

S. Weinberg, A model of leptons, Phys. Rev. Lett. 19 (1967) 1264;

A. Salam, Elementary particle theory: relativistic groups and analyticity, Nobel Symposium N8 Alquivist and Wilksells, Stockolm Sweden (1968);

S.L. Glashow, J. Iliopoulos and L. Maiani, Weak interactions with lepton-hadron symmetry, Phys. Rev. D 2 (1970) 1285.

[7] M.J. Ramsey-Musolf and S.A. Page, Hadronic parity violation: a new view through the looking glass, Ann. Rev. Nucl. Part. Sci. 56 (2006) 1 hep-ph/0601127;

E.G. Adelberg and W.C. Haxton, Parity violation in the nucleon-nucleon interaction, Ann. Rev. Nucl. Part. Sci. 35 (1985) 501;

B. Desplanques, J.F. Donoghue and B.R. Holstein, Unified treatment of the parity violating nuclear force, Ann. Phys. (NY) $\mathbf{1 2 4}(1980) 449$.

[8] S.L. Zhu, C.M. Maekawa, B.R. Holstein, M.J. Ramsey-Musolf and U. van Kolck, Nuclear parity-violation in effective field theory, Nucl. Phys. A 748 (2005) 435 [nucl-th/0407087; W.H. Hockings and U. van Kolck, The electric dipole form factor of the nucleon, Phys. Lett. B 605 (2005) 273 nucl-th/0508012.

[9] Z.G. Berezhiani, Grand unification of fermion masses, hep-ph/9312222.

[10] C. Hagedorn, M. Lindner and R.N. Mohapatra, $S_{4}$ flavor symmetry and fermion masses: towards a grand unified theory of flavor, JHEP 06 (2006) 042 hep-ph/0602244.

[11] M. Dress, R.M. Godbole and P. Royr, Theory and phenomenology of sparticles, 1st edition, World Scientific Publishing Co. Pte. Ltd., Singapore, (2004). 
[12] H. Baer and X. Tata, Weak scale supersymmetry, 1st edition, Cambridge University Press, Cambridge U.K. (2006).

[13] F. Gabbiani, E. Gabrielli, A. Masiero and L. Silvestrini, A complete analysis of FCNC and CP constraints in general SUSY extensions of the standard model, Nucl. Phys. B 477 (1996) 321 hep-ph/9604387;

M. Misiak, S. Pokorski and J. Rosiek, Supersymmetry and FCNC effects, Adv. Ser. Direct. High Energy Phys. 15 (1998) 795 hep-ph/9703442;

M. Ciuchini, E. Franco, A. Masiero and L. Silvestrini, $b \rightarrow s$ transitions: a new frontier for indirect SUSY searches, Phys. Rev. D 67 (2003) 075016 [Erratum ibid. D68 (2003) 079901] hep-ph/0212397.

[14] S. Weinberg, Mixing angle in renormalizable theories of weak and electromagnetic interactions, Phys. Rev. D 5 (1972) 1962.

[15] S. Weinberg, Electromagnetic and weak masses, Phys. Rev. Lett. 29 (1972) 388.

[16] L.E. Ibáñez, Hierarchical suppression of radiative quark and lepton masses in supersymmetric guts, Phys. Lett. B 117 (1982) 403.

[17] T. Banks, Supersymmetry and the quark mass matrix, Nucl. Phys. B 303 (1988) 172.

[18] E. Ma, Radiative quark and lepton masses through soft supersymmetry breaking, Phys. Rev. D 39 (1989) 1922.

[19] J. Ferrandis, Radiative mass generation and suppression of supersymmetric contributions to flavor changing processes, Phys. Rev. D 70 (2004) 055002 hep-ph/0404068.

[20] J. Ferrandis and N. Haba, Supersymmetry breaking as the origin of flavor, Phys. Rev. D 70 (2004) 055003 hep-ph/0404077.

[21] C.M. Maekawa and M.C. Rodriguez, Masses of fermions in supersymmetric models, JHEP 04 (2006) 031 hep-ph/0602074.

[22] H.P. Nilles, M. Olechowski and S. Pokorski, Does a radiative generation of quark masses provide us with the correct mass matrices?, Phys. Lett. B 248 (1990) 378.

[23] H.E. Haber and G.L. Kane, The search for supersymmetry: probing physics beyond the standard model, Phys. Rept. 117 (1985) 75.

[24] D. Bailin and A. Love, Supersymmetric gauge field theory and string theory, $\mathrm{\amalg OP}$, Bristol U.K. (1994).

[25] M.J. Ramsey-Musolf and S. Su, Low energy precision test of supersymmetry, hep-ph/0612057.

[26] D.J.H. Chung et al., The soft supersymmetry-breaking lagrangian: theory and applications, Phys. Rept. 407 (2005) 1 hep-ph/0312378.

[27] M. Frank and I. Turan, Rare decay of the top $t \rightarrow c l \bar{l}$ and single top production at international linear collider, Phys. Rev. D 74 (2006) 073014 hep-ph/0609069.

[28] A.M. Curiel, M.J. Herrero and D. Temes, Flavour changing neutral Higgs boson decays from squark: gluino loops, Phys. Rev. D 67 (2003) 075008 hep-ph/0210335.

[29] B.C. Allanach et al., The snowmass points and slopes: benchmarks for SUSY searches, Eur. Phys. J. C 25 (2002) 113 eConf C010630 (2001) P125, hep-ph/0202233;

http://spa.desy.de/spa. 
[30] J.A. Aguilar-Saavedra et al., Supersymmetry parameter analysis: SPA convention and project, Eur. Phys. J. C 46 (2006) 43 hep-ph/0511344.

[31] J.R. Ellis, K.A. Olive, Y. Santoso and V.C. Spanos, Very constrained minimal supersymmetric standard models, Phys. Rev. D 70 (2004) 055005 hep-ph/0405110.

[32] Particle Data Group collaboration, W.-M. Yao et al., Review of particle physics, J. Phys. G 33 (2006) 1.

[33] J.L. Diaz-Cruz, M. Gomez-Bock, R. Noriega-Papaqui and A. Rosado, A statistical analysis of the supersymmetric flavor problem and radiative fermion masses, hep-ph/0512168.

[34] S. Pokorski, Gauge field theories, 2nd edition, Cambridge University Press, Cambridge U.K. (2000).

[35] S. Davidson and M. Losada, Neutrino masses in the $R_{p}$ violating MSSM, JHEP 05 (2000) 021 hep-ph/0005080. 